\title{
Machine Learning in Economics and Finance
}

\author{
Periklis Gogas ${ }^{1} \cdot$ Theophilos Papadimitriou $^{1}$
}

Accepted: 8 January 2021 / Published online: 4 February 2021

(c) The Author(s), under exclusive licence to Springer Science+Business Media, LLC part of Springer Nature 2021

The term Machine Learning (ML) was introduced by Arthur Samuel while working for IBM in 1959, mainly to describe the pattern recognition tasks that delivered the "learning" component on the pioneering then Artificial Intelligence (AI) systems. The concept of Artificial Intelligence was theoretically and tentatively investigated from the 1930's though it was systematically studied after the famous Dartmouth Workshop of 1956 (Kline 2011). There, among other things, John McCarthy, research fellow at MIT at that time, proposed the term Artificial Intelligence over Cybernetics. In these early years, ML systems were only considered as part of a wider AI system. Since then, the range of practical applications of Machine Learning has been very wide, outreaching the narrow limits defined by the AI framework. Today, there are more autonomous ML systems than there are ML components in AI architectures. The terms AI and ML are often abusively interchanged for many reasons (trending, funding, or even ignorance), creating confusion to the non-expert. A general rule of thumb is that if the system acts without intervention, then it is probably AI. If the system classifies or forecasts through learning, then it is ML. The learning process was well established in 1997 by Professor Tom M. Mitchel from Carnegie Mellon University, in his famous quote from (1997) "A computer program is said to learn from experience $\mathrm{E}$ with respect to some class of tasks $\mathrm{T}$ and performance measure $\mathrm{P}$ if its performance at tasks in $\mathrm{T}$, as measured by $\mathrm{P}$, improves with experience E'. Self-driving cars use AI systems, the automatic vision system that identifies an imminent accident is ML.

During this scientific evolution, ML followed the fate of AI and experienced long periods of low interest and low funding, often referred to as "AI winters". Nonetheless, the present period is quite different as the timing of recent technological advances and the inception of new ML structures coincides ideally. Affordable parallel computing allowed the use of complex and demanding Deep Learning (DL) architectures like Recurrent Neural Networks and Convolutive Neural Networks to common applications. Moreover, algorithms such as Support Vector Machines and Random Forests and techniques such as kernelization, bagging and boosting allowed for the first time, the application of ML to relatively small datasets. In addition, the

Periklis Gogas

perrygogas@gmail.com

1 Department of Economics, Democritus University of Thrace, Komotini, Greece 
availability of free open-source Deep Learning libraries (supported by commercial giants) such as TensorFlow and PyTorch simplified the use of DL for everyone.

Machine Learning applied to economics problems can be traced back as early as 1974 (Lee and Lee 1974) although just as a mere mention in the abstract. The first paper that we came across actually applying a ML methodology exclusively on an economics problem is the study of Wang et al. (1984). The paper adopts the term AI, though it is most likely a misuse according to the distinction we made above, and the correct term should have been ML. In 1988 White, published a paper that involved a Neural Networks (NN) application to forecast the IBM daily stock returns. Since then, the appearance of ML in Economics steadily increased. Initially, it was applied in forecasting financial time series where long datasets are widely available. ML systems from that era required-for efficient training-extensive data sets that did not exist in other areas of economics. Furthermore, training was very time-consuming due to the-comparatively-low processing power of the computers of the period. Today, the use of many new ML architectures that do not require unreasonably long data sets, is an interesting and very promising avenue in Economic forecasting. This is the case not only for financial problems but also macroeconomics or microeconomics applications where data sets are inherently limited in size. As a result, recent ML applications in (for example) business cycles and recession forecasting seem very successful as compared to traditional empirical models. Currently, we encounter new methodologies merging and combining Econometrics with ML (i.e. Garch-SVM). Moreover, it is interesting how ML techniques and empirical procedures such as Cross Validation are now popular and are steadily adopted to traditional Econometrics methodologies.

The scope of this Special Issue was to publish state-of-the-art applications of Machine Learning in the areas of Economics and Finance. The innovation of the contributions lies either in the methodologies employed or the unique and innovative application of these algorithms that provide new and significant empirical insight in Economics. The Special Issue is composed of 17 papers with subjects ranging from forecasting economic and financial variables to simulating a complete stock market. Soybilgen and Yazgan tried to nowcast the US GDP growth, using tree-based models. In the same path, Yoon, forecasted the GDP growth of Japan using Random Forests and Gradient Boosting. Chen et al. used the GARCH model to identify the determinants of the Bitcoin price, and performed directional forecasting using Decision Trees and Support Vector Machines. Bouri et al. investigated the Bitcoin as a hedging choice, in the wake of the US-China trade war using Random Forests. Syriopoulos et al. tackled the problem of forecasting shipping prices using Support Vector Machines. Yilmaz and Arabaci conducted a thorough comparison between ten ML models on forecasting three exchange rates and concluded that the hybrid model fusing ARIMA and Long Short-Term Memory outperformed the competition. Similarly, Chakraborty et al. proposed a novel hybrid method combining ARIMA and Autoregressive NN to forecast the unemployment rate of seven countries. Plakandaras et al. forecast the price of Gold by combining the EEMD filtering method with the classic Support Vector Regression methodology. Following the trend of textual analysis, Lima et al., explores the forecasting ability of the textual features extracted from the FED minutes on the output growth. In the same 
vein, Duarte et al. combined the forecasting power of financial news and historical prices, to predict financial losses in the Brazilian stock market. A textual research of 1160 Computational Economics papers was performed by Alexakis et al. using the Latent Dirichlet Allocation, yielding 18 general topics of interest. Tsagris introduced a novel algorithm termed PCHC for Bayesian inference and used it in two cases: one involving credit card expenditures, and one the income level. Triebels et al. formulated the bank liquidity needs using three Recurrent Neural Networks architectures and data from the Mexican Banking Sector. The risk estimation of a $\mathrm{P} 2 \mathrm{P}$ lending market is investigated using a hybrid methodology combining InstanceBased Learning and Neural Networks in Babaei and Bambad. The same problem is investigated by Bussman et al. using Extreme Gradient Boosting and the Minimum Spanning Tree of the Shapley values. Pesantez-Narvaez et al. proposed the Synthetic Penalized Logitboost method based on weighting corrections to treat binary classification. They tested the proposed methodology against four other Econometrics and ML methods in the Home Mortgage Disclosure Act dataset. Lussange et al. created and tested a multiagent stock market simulator, where each stock acts autonomously using reinforcement learning.

We express our gratitude to all the researchers that trusted their studies in this Special Issue. The space of a journal issue, unfortunately, is relatively limited to cover all the submitted papers and we had to take some displeasing decisions. We must thank the Editor-in-Chief, Professor Hans M. Amman for accepting our proposal and helping us accomplish this guest editorship. We must also thank the valuable technical support provided by Naveen Parthiban.

\section{References}

Alexakis, C., Dowling, M., Eleftheriou, K., et al. (2020). Textual machine learning: An application to computational economics research. Computational Economics. https://doi.org/10.1007/s10614-02010077-3.

Babaei, G., \& Bambad, S. (2020). A new hybrid instance-based learning model for decision-making in the P2P lending market. Computational Economics. https://doi.org/10.1007/s10614-020-10085-3.

Bouri, E., Gkillas, K., Gupta, R., et al. (2020). Forecasting realized volatility of bitcoin: The role of the trade war. Computational Economics. https://doi.org/10.1007/s10614-020-10022-4.

Bussmann, N., Giudici, P., Marinelli, D., et al. (2020). Explainable machine learning in credit risk management. Computational Economics. https://doi.org/10.1007/s10614-020-10042-0.

Chakraborty, T., Chakraborty, A. K., Biswas, M., et al. (2020). Unemployment rate forecasting: A hybrid approach. Computational Economics. https://doi.org/10.1007/s10614-020-10040-2.

Chen, T. H., Chen, M. Y., \& Du, G. T. (2020). The determinants of bitcoin's price: Utilization of GARCH and machine learning approaches. Computational Economics. https://doi.org/10.1007/s10614-02010057-7.

Duarte, J. J., Montenegro González, S., \& Cruz, J. C. (2020). Predicting stock price falls using news data: Evidence from the Brazilian market. Computational Economics. https://doi.org/10.1007/s1061 4-020-10060-y.

Kline, R. R. (2011). Cybernetics, automata studies and the dartmouth conference on artificial intelligence. In IEEE Annals of the History of Computing, October-December. IEEE Computer Society.

Lee, S. C., \& Lee, E. (1974). Fuzzy sets and neural networks. Journal of Cybernetics, 4(2), 83-103. https ://doi.org/10.1080/01969727408546068. 
Lima, L. R., Godeiro, L. L., \& Mohsin, M. (2020). Time-varying dictionary and the predictive power of FED minutes. Computational Economics. https://doi.org/10.1007/s10614-020-10039-9.

Lussange, J., Lazarevich, I., Bourgeois-Gironde, S., et al. (2020). Modelling stock markets by multi-agent reinforcement learning. Computational Economics. https://doi.org/10.1007/s10614-020-10038-w.

Mitchell, T. (1997). Machine Learning (p. 2). New York: McGraw Hill. ISBN 978-0-07-042807-2

Pesantez-Narvaez, J., Guillen, M., \& Alcañiz, M. (2020). A synthetic penalized logitboost to model mortgage lending with imbalanced data. Computational Economics. https://doi.org/10.1007/s10614-02010059-5.

Plakandaras, V., Gogas, P., \& Papadimitriou, T. (2020). Gold against the machine. Computational Economics. https://doi.org/10.1007/s10614-020-10019-z.

Soybilgen, B., \& Yazgan, E. (2020). Nowcasting US GDP Using Tree-Based Ensemble Models and Dynamic Factors. Computational Economics. https://doi.org/10.1007/s10614-020-10083-5.

Syriopoulos, T., Tsatsaronis, M., \& Karamanos, I. (2020). Support vector machine algorithms: An application to ship price forecasting. Computational Economics. https://doi.org/10.1007/s10614-02010032-2.

Triepels, R., Daniels, H., \& Berndsen, R. (2020). Monitoring liquidity management of banks with recurrent neural networks. Computational Economics. https://doi.org/10.1007/s10614-020-10067-5.

Tsagris, M. (2020). A new scalable Bayesian network learning algorithm with applications to economics. Computational Economics. https://doi.org/10.1007/s10614-020-10065-7.

Wang, H., Li, C., Gu, B., \& Min, W. (1984). "Does AI-based credit scoring improve financial inclusion? Evidence from online payday lending". In 40th international conference on information systems. ICIS 2019.

White, H. (1988). Economic prediction using neural networks: The case of IBM daily stock returns. In IEEE 1988 international conference on neural networks, San Diego, CA, USA (vol. 2, pp. 451-458). https://doi.org/10.1109/ICNN.1988.23959.

Yilmaz, F. M., \& Arabaci, O. (2020). Should deep learning models be in high demand, or should they simply be a very hot topic? A comprehensive study for exchange rate forecasting. Computational Economics. https://doi.org/10.1007/s10614-020-10047-9.

Yoon, J. (2020). Forecasting of real GDP growth using machine learning models: Gradient boosting and random forest approach. Computational Economics. https://doi.org/10.1007/s10614-020-10054-w.

Publisher's Note Springer Nature remains neutral with regard to jurisdictional claims in published maps and institutional affiliations. 